\title{
LETTER \\ Computationally Efficient Cepstral Domain Feature Compensation
}

\author{
Woohyung LIM $^{\dagger \mathrm{a})}$, Chang Woo HAN ${ }^{\dagger \mathrm{b})}$, Nonmembers, and Nam Soo $\mathrm{KIM}^{\dagger \mathrm{c})}$, Member
}

SUMMARY In this letter, we propose a novel approach to feature compensation performed in the cepstral domain. Processing in the cepstral domain has the advantage that the spectral correlation among different frequencies is taken into consideration. By introducing a linear approximation with diagonal covariance assumption, we modify the conventional log-spectral domain feature compensation technique to fit to the cepstral domain. The proposed approach shows significant improvements in the AURORA2 speech recognition task.

key words: feature compensation, cepstral domain, linear approximation

\section{Introduction}

It is generally known that the performance degradation of a speech recognizer in the presence of background noise is one of the major obstacles to real-world application. In order to ameliorate the degradation in recognition performance in adverse environment, we can either suppress the noise in the feature domain or transform the models to match the input. There is also signal domain approach, such as the spectral subtraction algorithm, which, however, has been known to show poorer performance than both the feature domain and model domain techniques [1], [2]. In this letter, we focus on the feature compensation technique, in which the noisy input features are compensated before being decoded using the acoustic recognition models that were trained on clean speech. Since the proposed method provides not only the enhanced speech feature vectors but also the estimates for the noise, it can be easily extended to model adaptation.

Cepstrum is one of the predominant feature parameters for the state-of-the-art speech recognition systems because the cepstrum describes the speech characteristics in a very concise manner [3]. Extraction of the cepstra, however, requires a number of complex processes including nonlinear transforms such as the Mel-scale filter-bank, log function, and matrix operation such as the discrete cosine transform (DCT). For that reason, it is difficult to describe explicitly how the input speech cepstrum is affected by the cepstra of the clean speech and noise, which forces most of the feature compensation techniques to be performed in the log-spectral domain [4]-[7].

\footnotetext{
Manuscript received June 20, 2008.

Manuscript revised August 12, 2008

$\dagger$ The authors are with the School of Electrical Engineering and the Institute of New Media and Communications, Seoul National University, Seoul 151-742, Korea.

a)E-mail: whlim@hi.snu.ac.kr

b)E-mail: cwhan@hi.snu.ac.kr

c)E-mail: nkim@snu.ac.kr

DOI: 10.1587/transinf.E92.D.86
}

Even though the feature compensation in the logspectral domain is simple to implement, there are some disadvantages. One of them is the fact that the log-spectra need to be transformed into the corresponding cepstra by the DCT. Through DCT, the estimation errors in separate frequency bins of the log-spectrum are mixed together and make complicated artifacts which result in recognition performance degradation. In most of the feature compensation techniques, we usually assume that the log-spectral coefficients are independent of each other in order to simplify the computational model. However, actually the log-spectral coefficients are strongly correlated. For that reason, even though we try to make the estimation error minimized in the log-spectral domain, this does not guarantee the minimization of the error in the cepstral coefficients. On the other hand in the cepstral domain, since there is very weak correlation among different coefficients, the independence assumption can be more realistic. Therefore, to achieve a better performance in speech recognition, it is beneficial to compensate the cepstral coefficients directly [8].

In this letter, we propose a new feature compensation technique performed in the cepstral domain. We present a linear approximate method in the cepstral domain which enables us to easily adopt the conventional log-spectral domain feature compensation techniques in the cepstral domain. From a number of speech recognition experiments on AURORA2 database under the condition of clean training, it has been observed that the proposed approach improves the performance of the original interacting multiple model (IMM) technique, which is carried out in the log-spectral domain.

\section{Linear Approximation in Cepstral Domain}

In this section, let us consider how to approximate the speech corruption model in the cepstral domain. Let $s_{j}^{l}, n_{j}^{l}$ and $z_{j}^{l}$ denote the $j$ th log Mel-scale filter output of the clean speech, background noise and noisy speech, respectively, and $j=1,2, \ldots, N$. The superscript " $l$ " denotes that the relevant component is defined in the log-spectral domain. When the noise is added to the clean speech waveform, the relation in the log-spectral domain is given by

$$
z_{j}^{l}=\log \left(\exp \left(s_{j}^{l}\right)+\exp \left(n_{j}^{l}\right)\right) .
$$

In a similar way, we can obtain the speech corruption model in the cepstral domain by applying the DCT to (1) as follows: 


$$
\begin{aligned}
z_{i} & =\sum_{j=1}^{N} d_{i j} \log \left(\exp \left(s_{j}^{l}\right)+\exp \left(n_{j}^{l}\right)\right) \\
& =\sum_{j=1}^{N} d_{i j} \log \left(\exp \left(\sum_{k=1}^{N} e_{j k} s_{k}\right)+\exp \left(\sum_{k=1}^{N} e_{j k} n_{k}\right)\right)
\end{aligned}
$$

where $d_{i j}$ and $e_{i j}$ denote the $(i, j)$ th element of the $N \times N$ DCT matrix and the inverse DCT matrix, respectively. In (2), $s_{i}, n_{i}$ and $z_{i}$ denote the $i$ th cepstral coefficients of the clean speech, background noise and noisy speech, respectively. It should be noted that $z_{i}$ is related not only to the $i$ th cepstral coefficients of the clean speech and the background noise but also to the other coefficients. To compensate the $i$ th cepstral coefficient of the noisy speech, we should take all the cepstral coefficients of the clean speech and noise into consideration. This property makes it practically difficult to apply the feature compensation method to the cepstral coefficients directly.

To make the estimation problem a tractable one, we approximate (2) by a linear model given by

$$
z_{i} \approx \sum_{j=1}^{N} A_{i j}^{c}\left(s_{j}-s_{0, j}\right)+\sum_{j=1}^{N} B_{i j}^{c}\left(n_{j}-n_{0, j}\right)+C_{i}^{c}
$$

where $\left\{s_{0, j}, n_{0, j}\right\}$ are some fixed constants introduced for the convenience of formulation and $\left\{A_{i j}^{c}, B_{i j}^{c}, C_{i}^{c}\right\}$ are the constants which should be appropriately chosen. There are several methods for linear approximation such as the vector Taylor series (VTS) [9] and statistical linear approximation (SLA) [10]. In our work, apply the SLA method due to its superior performance.

When we use the first order SLA, $\left\{A_{i j}^{c}, B_{i j}^{c}, C_{i j}^{c}\right\}$ are given as follows [10]:

$$
\begin{aligned}
& A_{i j}^{c}=\left.\frac{\partial z_{i}}{\partial s_{j}}\right|_{s_{j}=s_{0, j}, n_{j}=n_{0, j}} \\
& B_{i j}^{c}=\left.\frac{\partial z_{i}}{\partial n_{j}}\right|_{s_{j}=s_{0, j}, n_{j}=n_{0, j}} \\
& C_{i}^{c}=\left.z_{i}\right|_{s_{j}=s_{0, j}, n_{j}=n_{0, j}} .
\end{aligned}
$$

After some algebra using (2) and (4), it can be shown that

$$
\begin{aligned}
A_{i j}^{c} & =\sum_{l=1}^{N} d_{i l} \frac{\exp \left(\sum_{k=1}^{N} e_{l k} s_{0, k}\right)}{\exp \left(\sum_{k=1}^{N} e_{l k} s_{0, k}\right)+\exp \left(\sum_{k=1}^{N} e_{l k} n_{0, k}\right)} e_{l j} \\
B_{i j}^{c} & =\sum_{l=1}^{N} d_{i l} \frac{\exp \left(\sum_{k=1}^{N} e_{l k} n_{0, k}\right)}{\exp \left(\sum_{k=1}^{N} e_{l k} s_{0, k}\right)+\exp \left(\sum_{k=1}^{N} e_{l k} n_{0, k}\right)} e_{l j} \\
C_{i}^{c} & =\sum_{l=1}^{N} d_{i l} \log \left(\exp \left(\sum_{k=1}^{N} e_{l k} s_{0, k}\right)+\exp \left(\sum_{k=1}^{N} e_{l k} n_{0, k}\right)\right) .
\end{aligned}
$$

In the case of using the second order SLA, $A_{i j}^{c}$ and $B_{i j}^{c}$ can be obtained in the same way to (4) while $C_{i j}^{c}$ is now modified to

$$
\begin{aligned}
C_{i}^{c}= & \left.z_{i}\right|_{s_{j}=s_{0, j}, n_{j}=n_{0, j}} \\
& +\left.\frac{1}{2} \frac{\partial^{2} z_{i}}{\partial s_{j}^{2}}\right|_{s_{j}=s_{0, j}, n_{j}=n_{0, j}} \cdot \operatorname{VAR}\left(s_{j}\right) \\
& +\left.\frac{1}{2} \frac{\partial^{2} z_{i}}{\partial n_{j}^{2}}\right|_{s_{j}=s_{0, j}, n_{j}=n_{0, j}} \cdot \operatorname{VAR}\left(n_{j}\right)
\end{aligned}
$$

where $\operatorname{VAR}(a)$ means the variance of the random variable $a$. For the derivation of (6), it is assumed that the cepstral coefficients possess the following correlation structure:

$$
\begin{aligned}
& \operatorname{COV}\left(s_{i}, s_{j}\right)=0, \text { for } i \neq j \\
& \operatorname{COV}\left(n_{i}, n_{j}\right)=0, \text { for } i \neq j \\
& \operatorname{COV}\left(s_{i}, n_{j}\right)=0, \text { for all } i, j
\end{aligned}
$$

with $\operatorname{COV}(a, b)$ denoting the covariance between the two random variables, $a$ and $b$. The validation of this assumption is given in Sect. 4.

From (2) and (6), we can obtain the linearization parameters for the second order SLA as follows:

$$
\begin{aligned}
A_{i j}^{c}= & \sum_{l=1}^{N} d_{i l} \frac{\exp \left(\sum_{k=1}^{N} e_{l k} s_{0, k}\right)}{\exp \left(\sum_{k=1}^{N} e_{l k} s_{0, k}\right)+\exp \left(\sum_{k=1}^{N} e_{l k} n_{0, k}\right)} e_{l j} \\
B_{i j}^{c}= & \sum_{l=1}^{N} d_{i l} \frac{\exp \left(\sum_{k=1}^{N} e_{l k} n_{0, k}\right)}{\exp \left(\sum_{k=1}^{N} e_{l k} s_{0, k}\right)+\exp \left(\sum_{k=1}^{N} e_{l k} n_{0, k}\right)} e_{l j} \\
C_{i}^{c}= & \sum_{l=1}^{N} d_{i l} \log \left(\exp \left(\sum_{k=1}^{N} e_{l k} s_{0, k}\right)+\exp \left(\sum_{k=1}^{N} e_{l k} n_{0, k}\right)\right) \\
& +\frac{1}{2} \sum_{l=1}^{N}\left[\sum_{j=1}^{N} d_{i j} \frac{\mathrm{e}^{\Sigma_{k=1}^{N} e_{j k} s_{0, k}} \mathrm{e}^{\Sigma_{k=1}^{N} e_{j k} n_{0, k}}}{\left(\mathrm{e}^{\Sigma_{k=1}^{N} e_{j k} s_{0, k}}+\mathrm{e}^{\Sigma_{k=1}^{N}} e_{j k} n_{0, k}\right)^{2}} e_{j l}^{2}\right] \\
& \cdot\left[V A R\left(s_{l}\right)+V A R\left(n_{l}\right)\right] .
\end{aligned}
$$

To apply (8) to the IMM feature compensation algorithm [7], we describe (3) in a vector-matrix form as follows:

$$
\mathbf{z} \approx \mathbf{f}(\mathbf{s}, \mathbf{n})=\mathbf{A}^{c}\left(\mathbf{s}-\mathbf{s}_{0}\right)+\mathbf{B}^{c}\left(\mathbf{n}-\mathbf{n}_{0}\right)+\mathbf{C}^{c}
$$

where $\mathbf{z}=\left[\begin{array}{llll}z_{1} & z_{2} & \ldots & z_{N}\end{array}\right]^{\prime}, \mathbf{s}=\left[\begin{array}{llll}s_{1} & s_{2} & \ldots & s_{N}\end{array}\right]^{\prime}$ and $\mathbf{n}=\left[\begin{array}{ll}n_{1} & \end{array}\right.$ $\left.n_{2} \ldots n_{N}\right]^{\prime}$ with the prime denoting matrix or vector transpose. In (9), $\mathbf{A}^{c}$ and $\mathbf{B}^{c}$ are matrices composed of $\left\{A_{i j}^{c}\right\}$ and $\left\{B_{i j}^{c}\right\}$, respectively, and $\mathbf{C}^{c}$ indicates the column vector $\left[C_{1}^{c}\right.$ $\left.C_{2}^{c} \ldots C_{N}^{c}\right]^{\prime}$. As mentioned above, $\mathbf{A}^{c}$ and $\mathbf{B}^{c}$ are $N \times N$ full matrices, which are not desirable for feature compensation using the IMM algorithm where inversion of the matrices $\mathbf{A}^{c}$ and $\mathbf{B}^{c}$ are needed to estimate the clean speech feature [7]. Therefore, it is necessary to make the matrices $\mathbf{A}^{c}$ and $\mathbf{B}^{c}$ structured in a simpler form. 


\section{Diagonally Structured Approximation}

In this section, we propose a modified linear approximation model in which the linear coefficient matrices are diagonally structured. Let $\{\mathbf{A}, \mathbf{B}, \mathbf{C}\}$ be matrices of the linearizing coefficients. Then the speech corruption model in the cepstral domain is approximated such that

$$
\mathbf{z} \approx \mathbf{g}(\mathbf{s}, \mathbf{n})=\mathbf{A}\left(\mathbf{s}-\mathbf{s}_{0}\right)+\mathbf{B}\left(\mathbf{n}-\mathbf{n}_{0}\right)+\mathbf{C} .
$$

Our purpose is to minimize the approximation error

$$
\begin{aligned}
e^{2} & =E\left[(\mathbf{g}(\mathbf{s}, \mathbf{n})-\mathbf{f}(\mathbf{s}, \mathbf{n}))^{2}\right] \\
& =\sum_{i=1}^{N} e_{i}^{2}
\end{aligned}
$$

where

$$
\begin{array}{r}
e_{i}^{2}=E\left[\left(\left(\sum_{j=1}^{N} A_{i j}^{c}\left(s_{j}-s_{0, j}\right)+\sum_{j=1}^{N} B_{i j}^{c}\left(n_{j}-n_{0, j}\right)+C_{i}^{c}\right)\right.\right. \\
\left.\left.-\left(A_{i}\left(s_{i}-s_{0, i}\right)+B_{i}\left(n_{i}-n_{0, i}\right)+C_{i}\right)\right)^{2}\right]
\end{array}
$$

and $A_{i}$ and $B_{i}$ denote the $(i, i)$ th coefficient of the diagonal matrices $\mathbf{A}$ and $\mathbf{B}$, respectively.

From (12) and (7), it can be straightforwardly proven that the optimal diagonal metrices are given by

$$
\begin{aligned}
A_{i} & =A_{i i}^{c} \\
B_{i} & =B_{i i}^{c} \\
C_{i} & =C_{i}^{c} .
\end{aligned}
$$

Using (13), we can establish the cepstral domain IMM algorithm in the same way as the conventional log-spectral domain technique [7]. Since $\mathbf{A}$ and $\mathbf{B}$ are diagonal, each cepstral coefficient can be compensated independently.

\section{Experimental Results}

Performance of the cepstral domain IMM (IMM-CEP) algorithm was evaluated on the AURORA2 database which consists of the TI-DIGITS data down-sampled to $8 \mathrm{kHz}$ [11]. The AURORA2 database is regarded as the clean speech data and it was artificially contaminated by adding the noises recorded under several conditions. Three sets of speech database were prepared for the recognition experiments. In test set A, the four noises (subway, babble, car and exhibition hall) were added to the clean data at signalto-noise ratio's (SNR's) of 20, 15, 10, 5, 0 and $-5 \mathrm{~dB}$. In test set B, another four types of noises (restaurant, street, airport and train station) were added to the clean data at the same SNR's. Finally in test set C, two of the noises from set A and set B (subway and street) were added to the clean data and there also existed a channel mismatch. Results are presented as an average performance in the five SNR conditions

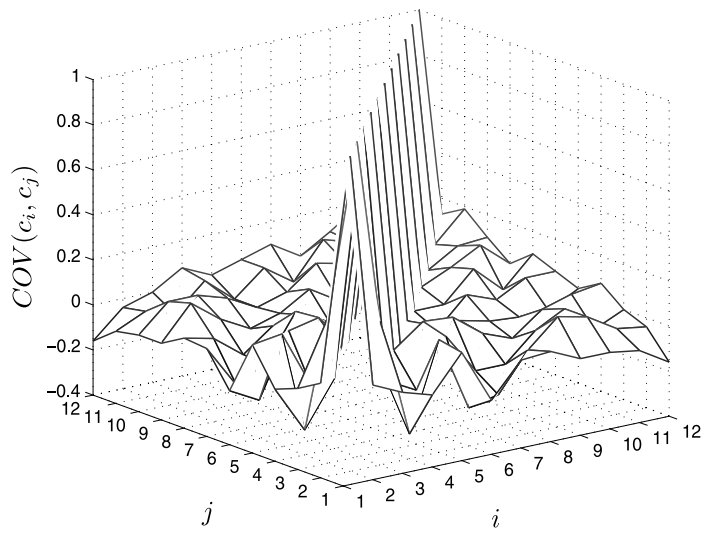

Fig. 1 Normalized covariances of cepstral coefficients (from clean speech training data).

Table 1 Word accuracies(\%) over AURORA2 database for clean training condition (relative improvements(\%) compared to the baseline system).

\begin{tabular}{c|c|ccc|c}
\hline & SNR & set A & set B & set C & Avg. \\
\hline \hline \multirow{5}{*}{ Baseline } & $20 \mathrm{~dB}$ & 94.84 & 92.45 & 96.00 & 94.11 \\
& $15 \mathrm{~dB}$ & 88.08 & 82.74 & 92.10 & 86.75 \\
& $10 \mathrm{~dB}$ & 71.01 & 62.84 & 81.40 & 69.82 \\
& $5 \mathrm{~dB}$ & 43.81 & 34.64 & 39.54 & 43.29 \\
& $0 \mathrm{~dB}$ & 19.79 & 14.29 & 30.08 & 19.64 \\
& Avg. & $\mathbf{6 3 . 5 0}$ & $\mathbf{5 7 . 3 9}$ & $\mathbf{7 1 . 8 2}$ & $\mathbf{6 2 . 7 2}$ \\
\hline \multirow{5}{*}{ IMM } & $20 \mathrm{~dB}$ & 97.30 & 96.99 & 96.27 & 96.97 \\
& $15 \mathrm{~dB}$ & 95.23 & 95.05 & 93.09 & 94.73 \\
& $10 \mathrm{~dB}$ & 90.37 & 90.24 & 86.16 & 89.47 \\
& $5 \mathrm{~dB}$ & 80.06 & 78.99 & 72.98 & 78.21 \\
& $0 \mathrm{~dB}$ & 55.80 & 55.99 & 46.34 & 53.98 \\
& Avg. & $\mathbf{8 3 . 7 5}$ & $\mathbf{8 3 . 4 5}$ & $\mathbf{7 8 . 9 7}$ & $\mathbf{8 2 . 6 7}$ \\
& & & & & $\mathbf{( 4 7 . 7 1 )}$ \\
\hline & $20 \mathrm{~dB}$ & 97.76 & 97.39 & 97.69 & 97.60 \\
& $15 \mathrm{~dB}$ & 96.02 & 95.77 & 95.05 & 95.73 \\
& $10 \mathrm{~dB}$ & 91.57 & 91.58 & 89.49 & 91.16 \\
& $5 \mathrm{~dB}$ & 81.88 & 81.06 & 77.44 & 80.66 \\
& $0 \mathrm{~dB}$ & 59.79 & 59.02 & 52.82 & 58.09 \\
& Avg. & $\mathbf{8 5 . 4 0}$ & $\mathbf{8 4 . 9 7}$ & $\mathbf{8 2 . 5 0}$ & $\mathbf{8 4 . 6 5}$ \\
& & & & & $\mathbf{( 5 6 . 6 8 )}$ \\
\hline
\end{tabular}

from $20 \mathrm{~dB}$ to $0 \mathrm{~dB}$.

Feature compensation was performed in the cepstral domain. In the proposed IMM-CEP algorithm, clean speech cepstra were modeled by a mixture of 128 Gaussian distributions with diagonal covariance matrices. We applied the second order SLA to linearize the speech corruption model in the cepstral domain.

In (7), we assume that cepstral coefficients are independent with each other. At first, it seems clear that the clean speech cepstra and the noise cepstra are independent. Secondly, the cepstral coefficients of the noise demonstrate weaker autocorrelations than those of the clean speech. To verify this, we pooled all the clean speech cepstra extracted from the Aurora 2 database and calculated the sample covariance matrix. The obtained normalized covariance matrix is shown in Fig. 1 where $c_{i}$ represents the $i$ th cepstral coefficient.

It is clear that each cepstral coefficient is almost completely uncorrelated with the other coefficients. Therefore 
the assumption that the cepstrum is independent of each other is validated to some extent experimentally.

The recognition results obtained from the AURORA2 task in clean training condition are shown in Table 1 where IMM-CEP denotes the proposed feature compensation technique performed directly in the cepstral domain and IMM denotes the conventional log-spectrum-based IMM technique [7]. The relative improvement represents the reduction rate in averaged word recognition error compared to the baseline over the SNR range from $20 \mathrm{~dB}$ to $0 \mathrm{~dB}$. In Table 1 , we can easily observe that the IMM-CEP approach outperformed the conventional IMM algorithm which was carried out in the log-spectral domain. There was a relatively more prominent performance improvement in set C. Although both of the IMM and the IMM-CEP algorithms are performed with prior knowledge on clean speech feature distribution, the cepstrum distribution is found more efficient than that of the log spectrum, not only in compensating the additive noise, but also in reducing the channel mismatch.

\section{Conclusions}

In this letter, we have presented a new feature compensation technique in the cepstral domain. We use the SLA method to linearize the non-linear relation among cepstral coefficients of the clean speech, noise and noisy speech. The coefficients of the linear approximation are designed to have a diagonal structure which enables us to apply the original IMM algorithm with little modification. From a number of experiments on the AURORA2 database, it has been discovered that the proposed approach outperforms the conventional IMM algorithm carried out in the log-spectral domain.

\section{Acknowledgments}

This work was supported in part by the Korea Science and
Engineering Foundation (KOSEF) grant funded by Korea government (MOST) (No.R0A-2007-000-10022-0) and by the Seoul R\&BD Program (10544).

\section{References}

[1] N.S. Kim, Y.J. Kim, and H.W. Kim, "Feature compensation based on soft decision,” IEEE Signal Process. Lett., vol.11, no.3, pp.378381, March 2004.

[2] B. Milner, J. Darch, and S. Vaseghi, "Applying noise compensation methods to robustly predict acoustic speech features from MFCC vectors in noise," Proc. ICASSP, pp.3945-3948, April 2008.

[3] S. Davis and P. Mermelstein, "Comparison of parametric representations for monosyllable word recognition in continuously spoken sentences," IEEE Trans. Acoust. Speech Signal Process., vol.28, no.4, pp.357-366, Aug. 1980.

[4] L. Deng, J. Droppo, and A. Acero, "A Bayesian approach to speech feature enhancement using the dynamic cepstral prior,' Proc. ICASSP, vol.1, pp.829-832, May 2002.

[5] L. Deng, J. Droppo, and A. Acero, "Enhancement of log Mel power spectra of speech using a phase-sensitive model of the acoustic environment and sequential estimation of the corrupting noise," IEEE Trans. Speech Audio Process., vol.12, no.2, pp.133-143, March 2004.

[6] N.S. Kim, "IMM-based estimation for slowly evolving environments," IEEE Signal Process. Lett., vol.5, no.6, pp.146-149, June 1998.

[7] N.S. Kim, "Feature domain compensation of nonstationary noise for robust speech recognition," Speech Commun., vol.37, no.4, pp.231248, July 2002.

[8] W. Lim, C.W. Han, J.W. Shin, and N.S. Kim, "Cepstral domain feature compensation based on diagonal approximation," Proc. ICASSP, pp.4401-4404, March 2008.

[9] P.J. Moreno, B. Raj, and R.M. Stern, "A vector Taylor series approach for environment-independent speech recognition," Proc. ICASSP, pp.733-736, May 1996.

[10] N.S. Kim, "Statistical linear approximation for environment compensation," IEEE Signal Process. Lett., vol.5, no.1, pp.8-10, Jan. 1998.

[11] H.-G. Hirsch and D. Pearch, "The aurora experimental framework for the performance evaluation of speech recognition systems under noisy conditions," Proc. ICSLP, pp.16-20, Oct. 2000. 